AZEVEDO AM; ANDRADE JÚNIOR VC; FERNANDES JSC. 2016. Transformação Box-Cox na homocedasticidade e normalidade uni e multivariada em experimentos de batata-doce. Horticultura Brasileira 34: 093-101. DOI - http://dx.doi.org/10.1590/S0102-053620160000100014

\title{
Transformação Box-Cox na homocedasticidade e normalidade uni e multivariada em experimentos de batata-doce
}

\author{
Alcinei M Azevedo'; Valter C Andrade Júnior²; José SC Fernandes² \\ ${ }^{1}$ Universidade Federal de Viçosa (UFV), Viçosa-MG, Brasil; alcineimistico@hotmail.com; ${ }^{2}$ Universidade Federal dos Vales do \\ Jequitinhonha e Mucuri (UFVJM), Diamantina-MG, Brasil; valterjr@ufvjm.edu.br; cunha.fernandes@ufvjm.edu.br
}

\begin{abstract}
RESUMO
A violação das pressuposições de normalidade e homocedasticidade dos resíduos pode ser um complicador nas provas de hipóteses e estimação de parâmetros. Quando não são satisfeitas, geralmente em consequência da escala de mensuração, a transformação desta escala pode levar ao atendimento destes pressupostos. Objetivou-se verificar o efeito da transformação Box-Cox na estimativa de parâmetros genéticos, homocedasticidade e normalidade uni e multivariada, baseando-se em onze experimentos de batata-doce. Os experimentos foram conduzidos no delineamento de blocos casualizados entre os anos de 2007 e 2011. A transformação Box-Cox foi estudada independente de haver ou não homocedasticidade e normalidade dos resíduos. As características produtividade total e comercial de raízes, massa média de raízes comerciais, produtividade de ramas, resistência a insetos de solo e formato das raízes foram avaliadas. Não houve uma transformação específica que fosse ideal para cada variável estudada, e, além disso, nem sempre foi necessário o uso da transformação de dados. A transformação Box-Cox melhorou a homocedasticidade e normalidade uni e multivariada dos resíduos, porém, nem sempre estas hipóteses deixaram de ser rejeitadas após a transformação. A transformação Box-Cox praticamente não alterou as estimativas de herdabilidade, índice de variação e as conclusões do teste F para o efeito de tratamento na maioria dos casos; no entanto, promoveu reduções nas estimativas do coeficiente de variação experimental e genético.
\end{abstract}

Palavras-chave: Ipomoea batatas, parâmetros genéticos, homogeneidade das variâncias, análise de variância.

\begin{abstract}
Box-Cox transformation in homoscedasticity and normality univariate and multivariate in experiments evaluating sweet potato
\end{abstract}

Violating assumptions of normality and homoscedasticity of the residuals can be a complicating factor in the hypothesis tests and parameter estimation. When assumptions are not met, usually as a consequence of the measurement scale, the transformation of this scale can lead the suitability of the presuppositions. This study aimed to verify the effect of the Box-Cox transformation in the estimation of genetic parameters, homoscedasticity and univariate and multivariate normality of the residuals, based on eleven experiments with sweet potatoes. The experiments were carried out in a randomized block design between the years 2007 and 2011. The Box-Cox transformation was studied independently of the existence of homoscedasticity and normality of the residuals. The traits total and marketable root yield, average weight of marketable roots, vines productivity, soil insect resistance and shape of the roots were evaluated. No specific processing was ideal for each variable, and furthermore, processing data was not always necessary. The Box-Cox transformation has improved homoscedasticity and univariate and multivariate normality, however, not always these hypothesis are no longer rejected after the transformation. The Box-Cox transformation practically did not change the estimates of heritability and variation indexes and the conclusions of $\mathrm{F}$ test for treatment effect in most cases, however, it led to reductions in the estimates of environmental and genetic variation coefficient.

Keywords: Ipomoea batatas, genetic parameters, homogeneity of variance, analysis of variance.

(Recebido para publicação em 25 de julho de 2014; aceito em 9 de setembro de 2015) (Received on July 25, 2014; accepted on September 9, 2015)

\begin{abstract}
A maioria das variáveis mensuradas em experimentos na área biológica ajusta-se à distribuição normal. Porém, quando isso não ocorre, em algumas situações, há a aproximação da distribuição normal com o aumento do tamanho amostral, conforme prevê o teorema do limite central (Resende, 2007). Devido à importância da distribuição normal, a maioria das técnicas estatísticas paramétricas se fundamentam na pressuposição de normalidade.
\end{abstract}

Quando cada variável resposta é analisada individualmente, a normalidade univariada é necessária para que os níveis de significância reais dos testes de hipóteses sejam próximos aos estabelecidos pelo pesquisador. Porém, nos casos em que variáveis são analisadas simultaneamente, as inferências estatísticas dependem da distribuição normal multivariada (Cantelmo \& Ferreira, 2007).

Além da normalidade, a homogenei- dade de variância, também denominada homocedasticidade, é muito importante para a inferência estatística (Couto et al., 2009), pois as hipóteses para os efeitos testáveis dos modelos estatístico são testadas considerando-se um resíduo comum. Segundo Resende (2007), a violação de qualquer uma das outras pressuposições da análise de variância (aditividade, independência e normalidade dos resíduos) pode conduzir à heterocedasticidade. 
Caso as pressuposições da normalidade e homocedasticidade não sejam atendidas, as informações obtidas pelas análises estatísticas podem gerar sérios equívocos (Xu et al., 2013). Em certos casos, quando não é possível satisfazer as pressuposições para análise de variância na própria escala observada, transformações têm sido feitas para uma escala mais apropriada com o objetivo de se conseguir homogeneidade de variâncias e uma distribuição aproximadamente normal (Couto et al., 2009; Custódio \& Barbin, 2009; Lúcio et al., 2010, 2011).

A escolha do tipo adequado de transformação não é trivial, pois matematicamente, existem inúmeras possibilidades e apenas o método da "tentativa e erro" não é o mais recomendado. Caso a escolha da transformação seja equivocada, pode-se ter mais problemas com a normalidade e homocedasticidade do que utilizando os dados originais. A família de transformações Box-Cox (Box \& Cox, 1964) é amplamente utilizada, pois permite identificar a melhor transformação, com base na utilização de um valor $\lambda$ (lambda) que maximize o estimador de máxima verossimilhança e minimize o resíduo (Chung et al., 2007; Lúcio et al., 2011). Esse método produz uma transformação da variável resposta (Y) tal que as pressuposições de homocedasticidade e normalidade dos resíduos sejam simultaneamente satisfeitas (Couto et al., 2009).

A transformação Box-Cox é utilizada em várias áreas do conhecimento, como na econometria (Yang et al., 2006), na avaliação de bovinos (Freitas et al., 2005), na estatística genômica (Yang et al., 2006), no estudo da qualidade da água (Freni \& Mannina, 2012), no estudo de solos contaminados (Meloun et al., 2005), entre outros. Recentemente este método tem sido aplicado em experimentos agronômicos, em culturas como abobrinha italiana, brócolis, pimentão e milho (Couto et al., 2009; Lúcio et al., 2010, 2011; Toebe \& Cargnelutti Filho, 2013). Contudo, são raros na literatura os trabalhos que avaliam o efeito da transformação Box-Cox na homocedasticidade, normalidade uni e multivariada dos resíduos e nas estimativas de parâmetros genéticos.
No Brasil é crescente o número de experimentos com a cultura da batata doce, objetivando explorar a grande variabilidade genética existente para a obtenção de genótipos resistentes a pragas e doenças (Marchese et al., 2010; Massaroto et al., 2010); com melhor qualidade nutricional (Andrade Júnior et al., 2012; Azevedo et al., 2014); maior densidade de raízes (Cardoso et al., 2007); maior teor de matéria seca e produção de biomassa (Gonçalves Neto et al., 2012); maior aptidão para produção de etanol (Gonçalves Neto et al., 2011); maior produção de raízes para alimentação humana (Cardoso et al., 2005; Azevedo et al., 2014), e maior produção de ramas para alimentação animal (Viana et al., 2011; Andrade Júnior et al., 2012). Porém, não há trabalhos na literatura que tenham como enfoque a verificação da homocedasticidade e normalidade uni e multivariada dos resíduos na cultura da batata-doce, bem como na aplicação de estratégias que amenizem a violação destas pressuposições.

Neste trabalho objetivou-se verificar o efeito da transformação Box-Cox nas estimativas de parâmetros genéticos, homocedasticidade e normalidade uni e multivariada em onze experimentos de batata-doce.

\section{MATERIAL E MÉTODOS}

Os dados são provenientes de 11 experimentos realizados com a cultura da batata-doce, com diferentes números de tratamentos (clones), todos em blocos ao acaso, conduzidos entre os anos 2005 e 2013 pelo Núcleo de Estudos em Olericultura da Universidade Federal dos Vales do Jequitinhonha e Mucuri (UFVJM), no município de Diamantina-MG (Tabela 1).

As áreas para o plantio foram preparadas efetuando-se uma aração e uma gradagem com posterior sulcamento do solo para a formação das leiras. As adubações de solo foram feitas considerando o resultado da análise química do solo e as recomendações para a cultura (Ribeiro et al., 1999); os demais tratos culturais foram realizados de acordo com o recomendado por Filgueira
(2008).

Neste estudo, considerou-se para a análise estatística as características produtividade total de raízes em t/ha (PT), produtividade de raízes comerciais em $\mathrm{t} / \mathrm{ha}$ (PC), massa média de raízes comerciais em grama (PMRC), produtividade de ramas em t/ha (PR), resistência das raízes a insetos de solo ( $\mathrm{RI})$ e formato das raízes (FORM).

A resistência das raízes a insetos de solo foi classificada por notas de 1 a 5 (Azevedo et al., 2002), sendo a nota $1=$ raízes livres de danos causados por insetos, com aspecto comercial desejável; $2=$ raízes com poucos danos, mas com presença de algumas galerias e furos nas raízes; $3=$ raízes com danos perceptíveis (presença de galeria e furos nas raízes em maior intensidade), com aspecto comercial prejudicado; 4= raízes com muitos danos, praticamente imprestáveis para comercialização (presença de muitas galerias, furos e início de apodrecimento); e nota 5= raízes totalmente imprestáveis para fins comerciais (repletas de galerias, furos e apodrecimento mais avançado). $\mathrm{O}$ formato da raiz foi avaliado atribuindo-se notas de 1 a 5 (Azevedo et al., 2002), sendo a nota $1=$ formato fusiforme, regular, sem veias ou qualquer tipo de rachaduras; 2 = formato considerado bom, próximo de fusiforme e com algumas veias; 3 = formato desuniforme, com veias e bastante irregular; 4= raízes muito grandes, com veias e rachaduras, indesejáveis comercialmente; e nota $5=$ raízes totalmente fora dos padrões comerciais, muito irregulares e deformadas, com muitas veias e rachaduras. Para as variáveis resistência das raízes a insetos de solo e formato das raízes, considerou-se para cada parcela a média das notas atribuídas por três avaliadores previamente treinados.

Para cada característica estudada em cada um dos experimentos realizou-se a transformação pela metodologia Box \& Cox (1964) para estabilizar ou reduzir a variabilidade existente e normalizar os resíduos. Este método foi estudado independente de haver ou não homocedasticidade e normalidade dos resíduos. Box \& Cox (1964) definiram a seguinte família de transformações: 


$$
\mathrm{y}^{(\lambda)}=\left\{\begin{array}{l}
\frac{\mathrm{y}^{\lambda}-1}{\lambda}, \lambda \neq 0 \\
\log y, \lambda=0
\end{array}\right.
$$

A equação (1) pode ser expressa pela transformação normalizada a seguir, onde o jacobiano $\mathrm{J}(\lambda, \mathrm{z})=1$.

$$
\mathrm{Z}^{(\lambda)}=\left\{\begin{array}{l}
\frac{\mathrm{y}^{\lambda}-1}{\lambda \mathrm{G}^{\lambda-1}}, \lambda \neq 0 \\
G \log y, \lambda=0
\end{array}\right.
$$

em que: $\lambda$ é um número real e $G$ é a média geométrica das observações da variável y.

Para a escolha da melhor potência $\lambda$, considerou-se valores no intervalo -5 a 5 em intervalos de 0,0625 unidades, sendo substituídos na expressão (2). Para o conjunto de dados transformados para cada valor de $\lambda$ realizou-se a análise de variância considerando o modelo: $\mathrm{y}_{\mathrm{ij}}=$ $\mu+t_{i}+b_{j}+e_{i j}$, em que: $y_{i j}=$ observação do i-ésimo tratamento na j-ésima repetição; $\mu=$ média geral; $t_{i}=$ efeito do i-ésimo tratamento; $b_{j}=$ efeito do $j$-ésimo bloco; $\mathrm{e} \mathrm{e}_{\mathrm{ij}}=$ efeito do erro experimental. Os efeitos dos tratamentos e dos blocos foram considerados como aleatórios.

Para a determinação do melhor valor de $\lambda$, foi utilizado o estimador de máxima verossimilhança $\left(L_{\max }\right)$, substituindo-se a soma de quadrados residual $\left(\mathrm{SQE}_{(\lambda)}\right)$ obtido para cada valor de $\lambda$ na expressão:

$$
L_{\max (\lambda)}=-\frac{n}{2} \ln \left(\frac{S Q E_{\lambda}}{n}\right)
$$

Para o estabelecimento do melhor valor de $\lambda$ verificou-se a situação onde o $L_{\max }$ é maximizado.

O algoritmo para a execução das análises de variâncias e obtenção do $L_{\max }$ e dos parâmetros genéticos foi implementado no programa Matlab (versão 8.1.0.604). O estudo da normalidade univariada dos resíduos foi feito antes e após a transformação, considerando-se as estimativas de curtose, simetria e o teste de Lilliefors. Para a homocedasticidade, utilizou-se o teste Bartlett. Para estes testes (homocedasticidade e normalidade univariada) utilizou-se o programa GENES (Cruz, 2013). Para o estudo da normalidade multivariada, antes e após a transformação em cada um dos experimentos, realizou-se o teste de Mardia (1970) para curtose e assimetria e o teste de Henze-Zirkler T (Mecklin, 2004). Para estes testes, utilizou-se o programa SAS, utilizando a macro multinorm (Truxillo, 2005).

Para verificar a influência da transformação Box-Cox nos parâmetros genéticos, estimou-se o coeficiente de variação ambiental (CVe), coeficiente de variação genético $(\mathrm{CVg})$, índice de variação (CVe/CVg) e herdabilidade no sentido amplo. Todos os estimadores destes parâmetros são apresentados por Cruz et al. (2012) e foram estimados com o auxílio do programa GENES (Cruz, 2013).

\section{RESULTADOS E DISCUSSÃO}

Pelos valores de $\lambda$ obtidos pelo método Box-Cox (Tabela 2) verificou-se que não há uma transformação específica que seja ideal para cada uma das variáveis estudadas nos vários experimentos conduzidos. Toebe \& Cargnelutti Filho (2013), avaliando 13 experimentos com a cultura do milho e, Lúcio et al. (2010), avaliando quatro experimentos de brócolis, verificaram resultados similares, encontrando para uma mesma característica valores diferentes de $\lambda$ em função dos vários experimentos conduzidos.
Isto mostra que nem sempre a escolha da melhor transformação é trivial. Porém, em algumas situações, mesmo antes de analisar os dados já se admite a necessidade de utilizar uma transformação específica; como exemplo pode-se citar: variáveis avaliadas por contagem que geralmente seguem distribuição Poisson (possui média igual à variância) quando é indicada a transformação raiz quadrada; variáveis expressas em porcentagem que geralmente seguem distribuição binomial, quando é indicada a transformação angular; e variáveis nas quais se verifica proporcionalidade entre média e variância, aproximando-se da distribuição exponencial, quando é recomendada a transformação logarítmica (Banzatto \& Kronka, 2013).

$$
\text { A expressão } \mathrm{Z}^{(\lambda)}=\frac{\mathrm{y}^{\lambda}-1}{\lambda} \text { para }
$$
$\lambda \neq 0$, e $\mathrm{Z}^{(\lambda)}=\ln y$ para $\lambda=0$ é equivalente a $\mathrm{Z}^{(\lambda)}=\mathrm{Y}^{\lambda}$ para $\lambda \neq 0$ e $\mathrm{Z}^{(\lambda)}=\ln \mathrm{y}$ para $\lambda=0$ quando a análise de variância é invariante com transformações lineares (Box \& Cox, 1964; Lúcio et al., 2011). Desta forma, para a característica produtividade total de raízes no experimento I conclui-se que não há necessidade de efetuar a transformação $\left(\mathrm{Y}^{1}\right)$. Para as características massa média de raízes comerciais e formato de raízes no experimento III

Tabela 1. Ano de execução, número de genótipos, número de repetições e locais de 11 experimentos de batata-doce conduzidos pelo núcleo de estudos em olericultura da UFVJM (year of execution, number of genotypes, number of replications and locations of 11 experiments of sweet potato carried out by UFVJM). Diamantina, UFVJM, 2015.

\begin{tabular}{lcccc}
\hline Ensaios & $\begin{array}{c}\text { Ano de } \\
\text { execução }\end{array}$ & $\begin{array}{c}\mathbf{N}^{\circ} \text { de } \\
\text { Genótipos }\end{array}$ & $\begin{array}{c}\mathbf{N}^{\circ} \text { de } \\
\text { repetições }\end{array}$ & Local \\
\hline I & 2005 & 12 & 4 & Diamantina-MG \\
II & 2005 & 12 & 4 & Diamantina-MG \\
III & 2007 & 6 & 3 & Diamantina-MG \\
IV & $2007 / 08$ & 65 & 3 & Diamantina-MG \\
V & 2009 & 12 & 4 & Diamantina-MG \\
VI & 2009 & 8 & 3 & Diamantina-MG \\
VII & 2009 & 8 & 3 & Faz. Forquilha \\
VIII & 2009 & 15 & 4 & Diamantina-MG \\
IX & $2010 / 11$ & 7 & 4 & Couto Magalhães de Minas-MG \\
X & $2010 / 11$ & 15 & 4 & Couto Magalhães de Minas-MG \\
XI & 2013 & 72 & 3 & Diamantina-MG \\
\hline
\end{tabular}

${ }^{1}$ Fazenda Forquilha: localizada no Distrito de Batatal, município de Diamantina-MG (Forquilha Farm: located in the District of Batatal, Diamantina, Minas Gerais State, Brazil). 
Tabela 2. Valores da potência lambda $(\lambda)$ obtidos pelo procedimento Box-Cox no estudo de seis características avaliadas em 11 experimentos de batata-doce \{values of lambda $(\lambda)$ obtained by the Box-Cox procedure in the study of six characteristics evaluated in 11 experiments of sweet potato . Diamantina, UFVJM, 2015.

\begin{tabular}{lrrrrrc}
\hline \multirow{2}{*}{ Experimentos } & \multicolumn{7}{c}{ Valores de $\lambda$} \\
\cline { 2 - 7 } & \multicolumn{1}{c}{ PT } & PC & PMRC & PR & RI & FORM \\
\hline I & 10,0000 & 0,9375 & $-0,2500$ & 0,3750 & $-0,3750$ & $-12,5000$ \\
II & 0,5000 & 0,6875 & $-0,6250$ & 0,4375 & $-0,5625$ & $-0,8125$ \\
III & 11,2500 & 0,6250 & $\mathrm{NT}^{1}$ & 0,0000 & $-0,6875$ & NT \\
IV & 0,3125 & 0,3750 & $-0,3750$ & 0,1875 & $-17,5000$ & 0,9375 \\
V & 0,6250 & 0,6250 & 0,0000 & 0,6250 & 11,5150 & 0,6875 \\
VI & 0,3750 & 0,3750 & $-0,1250$ & $-0,5625$ & 0,5625 & $-0,5000$ \\
VII & 0,1250 & 0,3125 & $-0,6250$ & 0,9375 & $-19,3750$ & $-0,3750$ \\
VIII & 0,6250 & 0,4375 & $-0,3750$ & 0,7500 & $-0,1875$ & 11,8750 \\
IX & 0,0625 & $-0,0625$ & -2 & 0,6875 & - & - \\
X & $-0,1250$ & 0,1250 & - & 0,6250 & - & - \\
XI & 0,3750 & 0,4375 & $-0,1875$ & 0,1250 & - & - \\
\hline
\end{tabular}

${ }^{1} \mathrm{NT}=$ Dados que não sofreram transformação por não haver um ponto de máximo no estimador de máxima verossimilhança dentro do intervalo de lambda estudado (-5 a 5) (data which were not processed because there is no maximum point of the maximum likelihood estimator in the lambda range). ${ }^{2}(-)$ variáveis não avaliadas no experimento (variables not measured in the experiment); $\mathrm{PT}=$ produtividade total de raízes, $\mathrm{t} / \mathrm{ha}$ (total yield of roots, $\mathrm{t}$ / ha) $\mathrm{PC}=$ produtividade de raízes comerciais, $\mathrm{t} / \mathrm{ha}$ (marketable yield of roots, $\mathrm{t} / \mathrm{ha}$ ); $\mathrm{PMRC}=$ massa média de raízes comerciais, gramas (average mass of marketable roots, grams); PR= produtividade de ramas (yield of vines); RI= resistência das raízes a insetos de solo (resistance of roots agains soil insects); FORM= formato das raízes (shape of roots).

não se verificou um ponto de máximo no estimador de máxima verossimilhança dentro do intervalo de lambda estudado (-5 a 5); logo, não se realizou transformação para estas características no experimento III. Verificou-se que nessas três situações houve distribuição normal e homogeneidade das variâncias, sendo dispensável o uso de transformação (Tabela 3).

Os experimentos em que foram observados os maiores problemas de normalidade e homogeneidade de variâncias residuais foram os de número IV e XI (Tabela 3). Nestes experimentos foram também testados os maiores números de tratamentos ( 65 e 72 genótipos), no qual o tamanho do bloco pode ter influenciado na qualidade dos experimentos, já que a variação ambiental dentro do bloco pode ter sido muito grande. Segundo Pimentel-Gomes (2009), quando o número de tratamentos é grande, ou há limitações que restringem o tamanho dos blocos, deve-se recorrer a delineamentos mais é dificultado (Cavalcante et al., 2003) podendo haver heterogeneidade na textura, densidade, umidade do solo e na incidência de pragas. Outra justificativa para os elevados coeficientes de variação na produção de raízes, é o fato destas estarem no subsolo, não sendo imediatamente visíveis, implicando na colheita incompleta de algumas parcelas (Azevedo et al., 2015). Resultados semelhantes para o alto coeficiente de variação na cultura da batata-doce em variáveis relacionadas às raízes também foram encontradas por Cavalcante et al. (2003, 2006), Cardoso et al. (2005), Andrade Júnior et al. (2009), Moreira et al. (2011), Azevedo et al. (2014, 2015).

De forma geral, após a transformação foi obtida maior homocedasticidade das variâncias e melhores valores para o teste Lilliefors, e simetria (Tabela 3). Os testes de curtose e simetria permitem inferir sobre a normalidade, sendo o coeficiente de simetria considerado mais poderoso em relação à curtose (Santos \& Ferreira, 2003).

Em algumas situações, verificou-se que a transformação foi eficiente para proporcionar o aceite da hipótese de normalidade e homogeneidade das variâncias quando estas hipóteses de nulidade eram rejeitadas antes da transformação. Em outros casos, a transformação foi eficiente para reduzir os problemas com a não normalidade e heterogeneidade das variâncias, mas as hipóteses de nulidade continuaram sendo rejeitadas, concordando com os resultados de Couto et al. (2009), Lúcio et al. (2010) e Toebe \& Cargnelutti Filho (2013). É válido lembrar que essas hipóteses não são tão restritivas quanto parecem ser, pois os testes de hipóteses são robustos em relação aos pequenos desvios de normalidade e homocedasticidade (Resende, 2007). Além disso, a normalidade geralmente não se verifica nos experimentos, pois o tamanho amostral avaliado frequentemente não é grande o suficiente para o atendimento desta pressuposição (Pimentel-Gomes, 2009).

Todas as transformações utilizadas acarretaram na redução da média dos dados, ocasionada pela mudança de escala (Tabela 4). Para o coeficiente de variação residual, após a transformação, verificaram-se reduções expressivas 
Tabela 3. Teste Lilliefors, teste Bartlett e estimativas de curtose e simetria em seis características avaliadas em 11 experimentos de batata-doce (Lilliefors test, Bartlett test and estimates of kurtosis and symmetry on six characteristics evaluated in 11 experiments with sweet potato). Diamantina, UFVJM, 2015.

\begin{tabular}{|c|c|c|c|c|c|c|c|c|c|c|c|c|c|}
\hline \multirow{2}{*}{ Exp. } & \multirow{2}{*}{ Testes } & \multicolumn{2}{|c|}{ PT } & \multicolumn{2}{|c|}{ PC } & \multicolumn{2}{|c|}{ PMRC } & \multicolumn{2}{|c|}{ PR } & \multicolumn{2}{|c|}{ RI } & \multicolumn{2}{|c|}{ FORM } \\
\hline & & NT & $\mathbf{T}$ & NT & $\mathbf{T}$ & NT & $\mathbf{T}$ & NT & $\mathbf{T}$ & NT & $\mathbf{T}$ & NT & $\mathbf{T}$ \\
\hline \multirow{4}{*}{ I } & Curtose & $2,50^{\mathrm{ns}}$ & $\mathrm{NT}^{1}$ & $1,97^{\mathrm{ns}}$ & $1,96^{\mathrm{ns}}$ & $2,57^{\mathrm{ns}}$ & $2,13^{\mathrm{ns}}$ & $3,43^{\mathrm{ns}}$ & $3,22^{\mathrm{ns}}$ & $4,31^{\mathrm{ns}}$ & $4,08^{\mathrm{ns}}$ & $4,61^{*}$ & $3,37^{\mathrm{ns}}$ \\
\hline & Simetria & $0,15^{\text {ns }}$ & NT & $0,26^{\text {ns }}$ & $0,25^{\mathrm{ns}}$ & $-0,27^{\mathrm{ns}}$ & $0,43^{\text {ns }}$ & $0,20^{\text {ns }}$ & $-0,15^{\text {ns }}$ & $0,35^{\mathrm{ns}}$ & $0,35^{\text {ns }}$ & $0,20^{\text {ns }}$ & $0,01^{\mathrm{ns}}$ \\
\hline & Lilliefors & $0,08^{\mathrm{ns}}$ & NT & $0,16^{* *}$ & $1,45^{*}$ & $0,06^{\mathrm{ns}}$ & $0,13^{\mathrm{ns}}$ & $0,08^{\text {ns }}$ & $0,04^{\mathrm{ns}}$ & $0,09^{\text {ns }}$ & $0,08^{\mathrm{ns}}$ & $0,10^{\text {ns }}$ & $0,07^{\text {ns }}$ \\
\hline & Teste Bartlett & $7,36^{\text {ns }}$ & NT & $3,36^{\text {ns }}$ & $3,40^{\text {ns }}$ & $9,35^{\text {ns }}$ & $5,61^{\mathrm{ns}}$ & $15,27^{\mathrm{ns}}$ & $11,61^{\mathrm{ns}}$ & $25,72^{* *}$ & $17,31^{\text {ns }}$ & $24,58^{*}$ & $16,93^{\text {ns }}$ \\
\hline \multirow{4}{*}{ II } & Curtose & $3,52^{\text {ns }}$ & $3,30^{\text {ns }}$ & $4,24^{\text {ns }}$ & $4,25^{\mathrm{ns}}$ & $4,02^{\text {ns }}$ & $3,65^{\text {ns }}$ & $4,44^{*}$ & $2,95^{\text {ns }}$ & $3,14^{\text {ns }}$ & $2,30^{\mathrm{ns}}$ & $3,72^{\text {ns }}$ & $3,28^{\text {ns }}$ \\
\hline & Simetria & $-0,18^{\mathrm{ns}}$ & $-0,41^{\mathrm{ns}}$ & $-0,22^{\mathrm{ns}}$ & $-0,41^{\mathrm{ns}}$ & $0,83^{*}$ & $-0,22^{\mathrm{ns}}$ & $0,83^{*}$ & $0,14^{\mathrm{ns}}$ & $0,49^{\text {ns }}$ & $-0,12^{\mathrm{ns}}$ & $0,63^{\text {ns }}$ & $-0,04^{\mathrm{ns}}$ \\
\hline & Lilliefors & $0,09^{\text {ns }}$ & $0,08^{\mathrm{ns}}$ & $0,06^{\text {ns }}$ & $0,11^{\mathrm{ns}}$ & $0,13^{\text {ns }}$ & $0,08^{\text {ns }}$ & $0,09^{\text {ns }}$ & $0,04^{\mathrm{ns}}$ & $0,09^{\mathrm{ns}}$ & $0,06^{\mathrm{ns}}$ & $0,07^{\mathrm{ns}}$ & $0,06^{\mathrm{ns}}$ \\
\hline & Teste Bartlett & $24,22^{*}$ & $22,56^{*}$ & $26,26^{* *}$ & $26,27^{* *}$ & $21,19^{*}$ & $11,32^{\text {ns }}$ & $13,12^{\text {ns }}$ & $8,50^{\text {ns }}$ & $6,99^{\text {ns }}$ & $3,76^{\mathrm{ns}}$ & $14,88^{\text {ns }}$ & $8,65^{\text {ns }}$ \\
\hline \multirow{4}{*}{ III } & Curtose & $2,25^{\mathrm{ns}}$ & $2,26^{\mathrm{ns}}$ & $3,75^{\text {ns }}$ & $4,15^{\mathrm{ns}}$ & $3,80^{\text {ns }}$ & NT & $3,42^{\text {ns }}$ & $2,84^{\mathrm{ns}}$ & $3,64^{\text {ns }}$ & $2,87^{\mathrm{ns}}$ & $4,10^{\text {ns }}$ & NT \\
\hline & Simetria & $0,16^{\mathrm{ns}}$ & $0,17^{\mathrm{ns}}$ & $-0,48^{\mathrm{ns}}$ & $-0,52^{\mathrm{ns}}$ & $-0,75^{\mathrm{ns}}$ & NT & $0,36^{\text {ns }}$ & $0,69^{\mathrm{ns}}$ & $0,48^{\mathrm{ns}}$ & $-0,19^{\mathrm{ns}}$ & $2,76^{\mathrm{ns}}$ & NT \\
\hline & Lilliefors & $0,09^{\text {ns }}$ & $0,14^{\mathrm{ns}}$ & $0,05^{\text {ns }}$ & $0,05^{\mathrm{ns}}$ & $0,08^{\text {ns }}$ & NT & $0,11^{\mathrm{ns}}$ & $0,12^{\mathrm{ns}}$ & $0,08^{\text {ns }}$ & $0,09^{\text {ns }}$ & $-0,56^{\mathrm{ns}}$ & NT \\
\hline & Teste Bartlett & $2,64^{\mathrm{ns}}$ & $2,64^{\mathrm{ns}}$ & $12,74^{*}$ & $8,83^{\text {ns }}$ & $10,30^{\text {ns }}$ & NT & $6,36^{\text {ns }}$ & $3,41^{\mathrm{ns}}$ & $11,11^{*}$ & $6,46^{\mathrm{ns}}$ & $0,09^{\text {ns }}$ & NT \\
\hline \multirow{4}{*}{ IV } & Curtose & $6,74^{* *}$ & $4,41^{* *}$ & $4,27^{* *}$ & $3,57^{\mathrm{ns}}$ & $3,37^{\mathrm{ns}}$ & $2,68^{\mathrm{ns}}$ & $9,08^{* *}$ & $4,09^{* *}$ & $4,34^{* *}$ & $2,67^{\mathrm{ns}}$ & $3,06^{\text {ns }}$ & $3,05^{\text {ns }}$ \\
\hline & Simetria & $0,32^{\text {ns }}$ & $-0,07^{\mathrm{ns}}$ & $0,13^{\mathrm{ns}}$ & $0,17^{\mathrm{ns}}$ & $0,27^{\mathrm{ns}}$ & $-0,11^{\mathrm{ns}}$ & $0,84^{* *}$ & $0,14^{\mathrm{ns}}$ & $0,92^{* *}$ & $-0,26^{\mathrm{ns}}$ & $-0,03^{\mathrm{ns}}$ & $-0,04^{\mathrm{ns}}$ \\
\hline & Lilliefors & $0,07^{*}$ & $0,04^{\mathrm{ns}}$ & $0,09^{* *}$ & $0,06^{\mathrm{ns}}$ & $0,05^{\mathrm{ns}}$ & $0,04^{\mathrm{ns}}$ & $0,11^{* *}$ & $0,06^{\mathrm{ns}}$ & $0,11^{* *}$ & $0,04^{\mathrm{ns}}$ & $0,04^{\mathrm{ns}}$ & $0,04^{\mathrm{ns}}$ \\
\hline & Teste Bartlett & $119,19^{* *}$ & $108,14^{* *}$ & $141,72^{* *}$ & $98,91^{* *}$ & $99,70^{* *}$ & $57,87^{\mathrm{ns}}$ & $179,69^{* *}$ & $98,83^{* *}$ & $101,32^{* *}$ & $53,44^{\text {ns }}$ & $57,30^{\text {ns }}$ & $57,19^{\text {ns }}$ \\
\hline \multirow{4}{*}{ V } & Curtose & $2,41^{\text {ns }}$ & $2,38^{\mathrm{ns}}$ & $0,01^{\text {ns }}$ & $-0,10^{\mathrm{ns}}$ & $5,58^{* *}$ & $4,15^{\mathrm{ns}}$ & $3,63^{\mathrm{ns}}$ & $3,41^{\mathrm{ns}}$ & $2,97^{\mathrm{ns}}$ & $2,94^{\mathrm{ns}}$ & $2,51^{\mathrm{ns}}$ & $12,36^{\text {ns }}$ \\
\hline & Simetria & $0,02^{\text {ns }}$ & $-0,09^{\text {ns }}$ & $2,76^{\text {ns }}$ & $2,59^{\mathrm{ns}}$ & $0,93^{* *}$ & $0,44^{\text {ns }}$ & $0,35^{\text {ns }}$ & $-0,05^{\text {ns }}$ & $-0,14^{\mathrm{ns}}$ & $-0,11^{\mathrm{ns}}$ & $0,29^{\text {ns }}$ & $2,44^{\text {ns }}$ \\
\hline & Lilliefors & $0,06^{\text {ns }}$ & $0,06^{\mathrm{ns}}$ & $0,07^{\mathrm{ns}}$ & $0,05^{\mathrm{ns}}$ & $0,09^{\text {ns }}$ & $0,09^{\text {ns }}$ & $0,08^{\mathrm{ns}}$ & $0,09^{\text {ns }}$ & $0,04^{\mathrm{ns}}$ & $0,03^{\text {ns }}$ & $0,11^{\text {ns }}$ & $0,20^{\text {ns }}$ \\
\hline & Teste Bartlett & $4,67^{\mathrm{ns}}$ & $4,34^{\mathrm{ns}}$ & $10,20^{\text {ns }}$ & $8,75^{\mathrm{ns}}$ & $15,05^{\mathrm{ns}}$ & $13,66^{\mathrm{ns}}$ & $9,83^{\text {ns }}$ & $9,72^{\text {ns }}$ & $14,08^{\mathrm{ns}}$ & $14,00^{\text {ns }}$ & $12,36^{\text {ns }}$ & $0,16^{\mathrm{ns}}$ \\
\hline \multirow{4}{*}{ VI } & Curtose & $3,13^{\text {ns }}$ & $3,25^{\text {ns }}$ & $2,62^{\text {ns }}$ & $2,36^{\mathrm{ns}}$ & $3,24^{\mathrm{ns}}$ & $3,21^{\mathrm{ns}}$ & $2,28^{\text {ns }}$ & $1,94^{\mathrm{ns}}$ & $2,46^{\text {ns }}$ & $2,43^{\text {ns }}$ & $3,61^{\text {ns }}$ & $3,67^{\text {ns }}$ \\
\hline & Simetria & $-0,25^{\mathrm{ns}}$ & $-0,41^{\text {ns }}$ & $0,38^{\text {ns }}$ & $0,16^{\mathrm{ns}}$ & $0,01^{\mathrm{ns}}$ & $0,26^{\mathrm{ns}}$ & $0,60^{\text {ns }}$ & $-0,33^{\text {ns }}$ & $-0,04^{\mathrm{ns}}$ & $-0,10^{\text {ns }}$ & $-0,08^{\mathrm{ns}}$ & $0,17^{\mathrm{ns}}$ \\
\hline & Lilliefors & $0,05^{\text {ns }}$ & $0,05^{\mathrm{ns}}$ & $0,16^{\text {ns }}$ & $0,07^{\mathrm{ns}}$ & $0,05^{\text {ns }}$ & $0,12^{\text {ns }}$ & $0,22^{* *}$ & $0,13^{\text {ns }}$ & $0,10^{\mathrm{ns}}$ & $0,09^{\text {ns }}$ & $0,06^{\text {ns }}$ & $0,06^{\mathrm{ns}}$ \\
\hline & Teste Bartlett & $6,00^{\text {ns }}$ & $5,49^{\text {ns }}$ & $3,57^{\mathrm{ns}}$ & $2,97^{\mathrm{ns}}$ & $8,41^{\text {ns }}$ & $6,05^{\mathrm{ns}}$ & $6,20^{\text {ns }}$ & $2,62^{\mathrm{ns}}$ & $4,48^{\text {ns }}$ & $4,50^{\text {ns }}$ & $8,63^{\text {ns }}$ & $10,23^{\text {ns }}$ \\
\hline \multirow{4}{*}{ VII } & Curtose & $3,37^{\text {ns }}$ & $2,20^{\mathrm{ns}}$ & $3,29^{\text {ns }}$ & $2,34^{\mathrm{ns}}$ & $4,49^{\text {ns }}$ & $2,96^{\mathrm{ns}}$ & $4,51^{\mathrm{ns}}$ & $4,45^{\mathrm{ns}}$ & $2,83^{\text {ns }}$ & $2,48^{\mathrm{ns}}$ & $4,85^{*}$ & $2,29^{\text {ns }}$ \\
\hline & Simetria & $0,64^{\mathrm{ns}}$ & $0,12^{\text {ns }}$ & $0,60^{\text {ns }}$ & $0,11^{\mathrm{ns}}$ & $0,91^{\mathrm{ns}}$ & $-0,18^{\mathrm{ns}}$ & $0,18^{\mathrm{ns}}$ & $0,13^{\mathrm{ns}}$ & $-0,41^{\mathrm{ns}}$ & $0,09^{\mathrm{ns}}$ & $1,07^{*}$ & $-0,49^{\mathrm{ns}}$ \\
\hline & Lilliefors & $0,16^{\text {ns }}$ & $0,09^{\mathrm{ns}}$ & $0,13^{\text {ns }}$ & $0,12^{\mathrm{ns}}$ & $0,09^{\text {ns }}$ & $0,10^{\mathrm{ns}}$ & $0,06^{\text {ns }}$ & $0,05^{\mathrm{ns}}$ & $0,09^{\text {ns }}$ & $0,08^{\mathrm{ns}}$ & $0,06^{\mathrm{ns}}$ & $0,11^{\mathrm{ns}}$ \\
\hline & Teste Bartlett & $6,56^{\text {ns }}$ & $3,15^{\text {ns }}$ & $6,66^{\text {ns }}$ & $0,28^{\mathrm{ns}}$ & $11,60^{\text {ns }}$ & $8,47^{\mathrm{ns}}$ & $12,33^{\text {ns }}$ & $12,44^{\mathrm{ns}}$ & $5,44^{\text {ns }}$ & $4,21^{\mathrm{ns}}$ & $8,90^{\text {ns }}$ & $4,64^{\mathrm{ns}}$ \\
\hline \multirow{4}{*}{ VIII } & Curtose & $3,75^{\text {ns }}$ & $4,19^{*}$ & $3,76^{\text {ns }}$ & $3,56^{\mathrm{ns}}$ & $2,79^{\text {ns }}$ & $2,61^{\mathrm{ns}}$ & $3,04^{\mathrm{ns}}$ & $3,04^{\mathrm{ns}}$ & $2,60^{\mathrm{ns}}$ & $6,04^{\mathrm{ns}}$ & $3,39^{\text {ns }}$ & $3,37^{\mathrm{ns}}$ \\
\hline & Simetria & $0,75^{*}$ & $0,53^{\mathrm{ns}}$ & $0,60^{*}$ & $0,09^{\mathrm{ns}}$ & $0,22^{\mathrm{ns}}$ & $0,13^{\mathrm{ns}}$ & $0,09^{\text {ns }}$ & $-0,02^{\mathrm{ns}}$ & $0,04^{\mathrm{ns}}$ & $2,32^{\mathrm{ns}}$ & $0,45^{\text {ns }}$ & $0,47^{\mathrm{ns}}$ \\
\hline & Lilliefors & $0,17^{* *}$ & $0,10^{\mathrm{ns}}$ & $0,12^{*}$ & $0,06^{\mathrm{ns}}$ & $0,13^{*}$ & $0,07^{\mathrm{ns}}$ & $0,06^{\text {ns }}$ & $0,05^{\mathrm{ns}}$ & $0,07^{\mathrm{ns}}$ & $0,08^{\mathrm{ns}}$ & $0,11^{\mathrm{ns}}$ & $0,13^{*}$ \\
\hline & Teste Bartlett & $29,61^{* *}$ & $29,83^{* *}$ & $25,53^{*}$ & $22,20^{\text {ns }}$ & $11,81^{\mathrm{ns}}$ & $7,36^{\mathrm{ns}}$ & $32,48^{* *}$ & $35,70^{*}$ & $10,40^{\mathrm{ns}}$ & $0,09^{\text {ns }}$ & $46,74^{* *}$ & $49,90^{* *}$ \\
\hline \multirow{4}{*}{ IV } & Curtose & $7,66^{* *}$ & $2,22^{\mathrm{ns}}$ & $8,54^{* *}$ & $2,19^{\mathrm{ns}}$ & -2 & - & $2,78^{\mathrm{ns}}$ & $2,84^{\mathrm{ns}}$ & - & - & - & - \\
\hline & Simetria & $1,43^{* *}$ & $0,26^{\mathrm{ns}}$ & $1,43^{* *}$ & $-0,39^{\mathrm{ns}}$ & - & - & $-0,12^{\mathrm{ns}}$ & $-0,29^{\text {ns }}$ & - & - & - & - \\
\hline & Lilliefors & $0,21^{* *}$ & $0,15^{\mathrm{ns}}$ & $0,18^{*}$ & $0,13^{\mathrm{ns}}$ & - & - & $0,06^{\mathrm{ns}}$ & $0,07^{\mathrm{ns}}$ & - & - & - & - \\
\hline & Teste Bartlett & $16,56^{*}$ & $2,66^{\mathrm{ns}}$ & $17,21^{* *}$ & $7,54^{\mathrm{ns}}$ & - & - & $3,78^{\mathrm{ns}}$ & $3,99^{\text {ns }}$ & - & - & - & - \\
\hline \multirow{4}{*}{$X$} & Curtose & $4,68^{* *}$ & $3,01^{\text {ns }}$ & $6,21^{* *}$ & $2,60^{\mathrm{ns}}$ & - & - & $2,50^{\text {ns }}$ & $2,25^{\mathrm{ns}}$ & - & - & - & - \\
\hline & Simetria & $1,22^{* *}$ & $-0,42^{\text {ns }}$ & $1,31^{* *}$ & $0,17^{\mathrm{ns}}$ & - & - & $0,38^{\text {ns }}$ & $0,28^{\mathrm{ns}}$ & - & - & - & - \\
\hline & Lilliefors & $0,19^{* *}$ & $0,06^{\mathrm{ns}}$ & $0,12^{*}$ & $0,08^{\mathrm{ns}}$ & - & - & $0,09^{\text {ns }}$ & $0,10^{\text {ns }}$ & - & - & - & - \\
\hline & Teste Bartlett & $34,07^{* *}$ & $17,96^{\mathrm{ns}}$ & $23,36^{\text {ns }}$ & $7,35^{\mathrm{ns}}$ & $\therefore$ & - & $12,22^{\text {ns }}$ & $11,82^{\mathrm{ns}}$ & $\therefore$ & - & $\therefore$ & - \\
\hline \multirow{4}{*}{$\mathrm{XI}$} & Curtose & $7,05^{* *}$ & $3,61^{*}$ & $8,03^{* *}$ & $4,31^{* *}$ & $7,14^{* * *}$ & $4,53^{* *}$ & $5,21^{* * *}$ & $5,59^{* * *}$ & - & - & - & - \\
\hline & Simetria & $0,46^{* *}$ & $-0,04^{\mathrm{ns}}$ & $0,54^{* *}$ & $-0,13^{\mathrm{ns}}$ & $1,02^{* *}$ & $-0,03^{\mathrm{ns}}$ & $0,38^{* *}$ & $-0,56^{* *}$ & - & - & - & - \\
\hline & Lilliefors & $0,04^{\mathrm{ns}}$ & $0,03^{\mathrm{ns}}$ & $0,05^{\text {ns }}$ & $0,06^{*}$ & $0,06^{*}$ & $0,04^{\mathrm{ns}}$ & $0,06^{*}$ & $0,02^{\mathrm{ns}}$ & - & - & - & - \\
\hline & Teste Bartlett & $112,90^{* *}$ & $81,19^{\text {ns }}$ & $162,32^{* *}$ & $115,81^{* *}$ & $146,02^{* *}$ & $98,91^{*}$ & $142,68^{* *}$ & $99,28^{*}$ & - & - & - & - \\
\hline
\end{tabular}

${ }^{* *},{ }^{*} \mathrm{e}^{\text {ns }}$ referem-se à significância a $1 \%, 5 \%$ e não significativo a $5 \%$ respectivamente, pelo teste t para o coeficiente de simetria e curtose, pelo teste de Lilliefors e pelo teste $\mathrm{X}^{2}$ para o teste Bartlett (refer to significance considering $1 \%, 5 \%$ and not significant at the $5 \%$ respectively, for the coefficient $t$ by symmetry and kurtosis at Lilliefors test and the $\mathrm{X}^{2}$ test for the Bartlett's test). ${ }^{1}(\mathrm{NT})$ Dados que não sofreram transformação (data which were not transformed). ${ }^{2}(-)$ variáveis não avaliadas no experimento (variables not measured in the experiment); $\mathrm{PT}=$ produtividade total de raízes, $\mathrm{t} / \mathrm{ha}$ (total yield of roots, $\mathrm{t} / \mathrm{ha}$ ); $\mathrm{PC}=$ produtividade de raízes comerciais, $\mathrm{t} / \mathrm{ha}$ (yield of marketable roots, $\mathrm{t} / \mathrm{ha}$ ); $\mathrm{PMRC}=$ massa média de raízes comerciais, gramas (average mass of marketable roots, grams); $\mathrm{PR}=$ produtividade de ramas (yield of vines); RI= resistência das raízes a insetos de solo (resistance of roots agains soil insects); FORM= formato das raízes (shape of roots). 
Tabela 4. Média fenotípica, coeficiente de variação ambiental (CVe), coeficiente de variação genético (CVg), índice de variação genotípico (Iv) e herdabilidade no sentido amplo $\left(\mathrm{h}^{2}\right)$ e p-valor do efeito de tratamento pelo teste $\mathrm{F}(\mathrm{pV})$ em seis características avaliadas em 11 experimentos de batata-doce para os dados não transformados (NT) e para os dados transformados (T) \{mean phenotypic, environmental coefficient of variation $(\mathrm{CVe})$, coefficient of genetic variation $(\mathrm{CVg})$, genotypic variation index (Iv), broad sense heritability $\left(\mathrm{h}^{2}\right)$ and $\mathrm{p}$-value of the treatment effect by the test $\mathrm{F}(\mathrm{pV})$ on six characteristics evaluated in 11 experiments of sweet potato for no transformed data (NT) and transformed data $(\mathrm{T})\}$. Diamantina, UFVJM, 2015.

\begin{tabular}{|c|c|c|c|c|c|c|c|c|c|c|c|c|c|}
\hline \multirow{2}{*}{ Exp. } & \multirow{2}{*}{ Parâmetro } & \multicolumn{2}{|c|}{ PT } & \multicolumn{2}{|c|}{ PC } & \multicolumn{2}{|c|}{ PMRC } & \multicolumn{2}{|c|}{ PR } & \multicolumn{2}{|c|}{ RI } & \multicolumn{2}{|c|}{ FORM } \\
\hline & & NT & $\mathbf{T}$ & NT & $T$ & NT & $\mathbf{T}$ & NT & $T$ & NT & $T$ & NT & $\mathbf{T}$ \\
\hline \multirow{6}{*}{ I } & Média & 18,36 & NT & 14,98 & 12,58 & 256,71 & 0,25 & 12,55 & 2,52 & 1,79 & 0,82 & 2,11 & 0,42 \\
\hline & Cve (\%) & 21,91 & NT & 25,64 & 24,29 & 14,05 & 3,40 & 30,18 & 11,62 & 18,01 & 6,17 & 21,84 & 22,81 \\
\hline & CVg $(\%)$ & 28,89 & NT & 33,65 & 31,73 & 14,65 & 3,60 & 25,84 & 10,87 & 22,06 & 8,21 & 8,88 & 8,83 \\
\hline & Iv & 1,32 & NT & 1,31 & 1,31 & 1,04 & 1,06 & 0,86 & 0,93 & 1,22 & 1,33 & 0,41 & 0,39 \\
\hline & $h^{2}$ & 87,43 & NT & 87,32 & 87,23 & 81,30 & 81,70 & 74,57 & 77,76 & 85,72 & 87,64 & 39,79 & 37,48 \\
\hline & $\mathrm{pV}(\%)$ & $<0,01$ & NT & $<0,01$ & $<0,01$ & $<0,01$ & $<0,01$ & $<0,01$ & $<0,01$ & $<0,01$ & $<0,01$ & 12,72 & 14,48 \\
\hline \multirow{6}{*}{ II } & Média & 26,85 & 5,08 & 23,29 & 8,54 & 232,69 & 0,03 & 7,04 & 2,24 & 1,56 & 0,80 & 1,78 & 0,67 \\
\hline & Cve (\%) & 28,84 & 14,81 & 31,29 & 22,28 & 18,37 & 13,65 & 45,79 & 20,37 & 18,31 & 9,49 & 26,45 & 18,12 \\
\hline & CVg $(\%)$ & 25,45 & 12,67 & 25,44 & 17,72 & 16,14 & 12,16 & 39,55 & 19,55 & 15,58 & 8,21 & 18,97 & 16,64 \\
\hline & Iv & 0,88 & 0,85 & 0,81 & 0,80 & 0,88 & 0,89 & 0,86 & 0,96 & 0,85 & 0,86 & 0,72 & 0,92 \\
\hline & $h^{2}$ & 75,70 & 74,51 & 72,56 & 71,68 & 75,54 & 76,07 & 74,89 & 78,64 & 74,34 & 74,94 & 67,28 & 77,14 \\
\hline & $\mathrm{pV}(\%)$ & 0,11 & 0,08 & 0,19 & 0,24 & 0,08 & 0,11 & 0,10 & 0,03 & 0,12 & 0,10 & 0,63 & 0,05 \\
\hline \multirow{6}{*}{ III } & Média & 21,84 & 32,34 & 16,30 & 5,58 & 199,89 & $\mathrm{NT}^{1}$ & 7,93 & 0,80 & 1,78 & 0,69 & 2,24 & NT \\
\hline & Cve (\%) & 11,38 & 12,84 & 14,89 & 9,63 & 6,46 & NT & 25,75 & 11,36 & 12,22 & 7,23 & 9,19 & NT \\
\hline & CVg $(\%)$ & 26,76 & 29,97 & 38,83 & 28,85 & 7,60 & NT & 57,70 & 37,57 & 19,76 & 12,81 & 5,85 & NT \\
\hline & Iv & 2,35 & 2,30 & 2,61 & 3,00 & 1,18 & NT & 2,24 & 3,31 & 1,62 & 1,77 & 0,64 & NT \\
\hline & $h^{2}$ & 94,32 & 94,05 & 95,33 & 96,42 & 80,62 & NT & 93,78 & 97,04 & 88,69 & 90,41 & 54,86 & NT \\
\hline & $\mathrm{pV}(\%)$ & $<0,01$ & $<0,01$ & $<0,01$ & $<0,01$ & 6,46 & NT & $<0,02$ & $<0,01$ & 0,02 & 0,10 & 13,31 & NT \\
\hline \multirow{6}{*}{ IV } & Média & 10,33 & 2,03 & 6,02 & 1,88 & 162,22 & 0,15 & 3,20 & 1,20 & 1,52 & 0,55 & 3,16 & 3,08 \\
\hline & Cve (\%) & 34,85 & 10,82 & 41,29 & 15,96 & 25,28 & 8,91 & 59,88 & 10,32 & 24,33 & 31,64 & 23,00 & 22,58 \\
\hline & CVg (\%) & 31,14 & 9,95 & 46,10 & 17,11 & 13,31 & 4,63 & 41,77 & 9,48 & 8,59 & 15,39 & 19,98 & 19,63 \\
\hline & Iv & 0,89 & 0,92 & 1,12 & 1,07 & 0,53 & 0,52 & 0,70 & 0,92 & 0,35 & 0,49 & 0,87 & 0,87 \\
\hline & $\mathrm{h}^{2}$ & 70,55 & 71,76 & 78,90 & 77,51 & 45,42 & 44,74 & 59,34 & 71,70 & 27,20 & 41,50 & 69,35 & 69,39 \\
\hline & pV (\%) & $<0,01$ & $<0,01$ & $<0,01$ & $<0,01$ & 0,16 & 0,25 & $<0,01$ & $<0,01$ & 6,56 & 0,53 & $<0,01$ & $<0,01$ \\
\hline \multirow{6}{*}{ V } & Média & 25,00 & 7,30 & 20,88 & 6,48 & 353,91 & 2,53 & 18,49 & 6,03 & 2,01 & 2,25 & 2,03 & 1,62 \\
\hline & Cve (\%) & 37,60 & 24,71 & 41,36 & 27,38 & 25,20 & 4,21 & 41,28 & 27,09 & 16,25 & 18,77 & 17,78 & 12,31 \\
\hline & $\operatorname{CVg}(\%)$ & 19,39 & 12,90 & 21,69 & 14,59 & 11,92 & 2,01 & 17,19 & 14,14 & 21,32 & 23,98 & 8,17 & 5,57 \\
\hline & Iv & 0,52 & 0,52 & 0,52 & 0,53 & 0,47 & 0,48 & 0,42 & 0,52 & 1,31 & 1,28 & 0,46 & 0,45 \\
\hline & $\mathrm{h}^{2}$ & 51,56 & 52,16 & 52,38 & 53,18 & 47,23 & 47,67 & 40,96 & 52,15 & 87,32 & 86,72 & 45,78 & 45,07 \\
\hline & $\mathrm{pV}(\%)$ & 5,32 & 5,03 & 4,93 & 4,56 & 7,69 & 7,43 & 11,86 & 5,04 & $<0,01$ & $<0,01$ & 8,58 & 9,03 \\
\hline \multirow{6}{*}{ VI } & Média & 10,50 & 2,37 & 8,40 & 2,16 & 266,42 & 0,50 & 9,09 & 0,31 & 2,10 & 1,51 & 1,53 & 0,85 \\
\hline & Cve (\%) & 39,89 & 15,62 & 44,47 & 17,36 & 32,53 & 4,03 & 44,78 & 22,18 & 26,17 & 15,02 & 26,53 & 12,86 \\
\hline & CVg $(\%)$ & 13,27 & 4,91 & 8,83 & 4,36 & 17,39 & 3,09 & $\mathrm{X}^{2}$ & 5,46 & 10,71 & 5,65 & 10,71 & 12,96 \\
\hline & Iv & 0,33 & 0,31 & 0,20 & 0,25 & 0,53 & 0,77 & $X$ & 0,25 & 0,41 & 0,38 & 0,41 & 1,01 \\
\hline & $h^{2}$ & 24,92 & 22,89 & 10,58 & 15,92 & 46,16 & 63,71 & $X$ & 15,40 & 33,47 & 29,82 & 33,47 & 75,28 \\
\hline & $\mathrm{pV}(\%)$ & 30,61 & 32,06 & 40,47 & 36,91 & 15,35 & 5,05 & 54,43 & 37,26 & 24,42 & 27,08 & 0,58 & 1,26 \\
\hline \multirow{6}{*}{ VII } & Média & 25,03 & 1,47 & 22,67 & 2,56 & 360,35 & 0,03 & 50,36 & 39,31 & 1,41 & 0,63 & 2,27 & 0,75 \\
\hline & Cve (\%) & 50,92 & 6,33 & 52,49 & 16,81 & 26,25 & 12,77 & 26,30 & 24,81 & 19,20 & 23,69 & 13,88 & 4,30 \\
\hline & CVg $(\%)$ & 18,98 & 3,17 & 23,27 & 8,74 & 19,17 & 11,64 & 12,05 & 11,34 & 23,69 & 38,83 & 29,87 & 10,45 \\
\hline & Iv & 0,37 & 0,50 & 0,44 & 0,52 & 0,73 & 0,91 & 0,46 & 0,46 & 1,23 & 1,64 & 2,15 & 2,43 \\
\hline & $\mathrm{h}^{2}$ & 29,43 & 43,00 & 37,09 & 44,79 & 61,54 & 71,35 & 38,62 & 38,54 & 82,03 & 88,97 & 93,29 & 94,66 \\
\hline & $\mathrm{pV}(\%)$ & 27,36 & 17,55 & 21,79 & 16,3 & 6,07 & 2,22 & 20,68 & 20,74 & 0,32 & 0,03 & $<0,01$ & $<0,01$ \\
\hline \multirow{6}{*}{ VIII } & Média & 8,34 & 3,64 & 6,37 & 2,17 & 199,72 & 0,14 & 6,77 & 4,01 & 1,77 & 0,91 & 2,04 & 2,35 \\
\hline & Cve (\%) & 41,56 & 27,31 & 43,10 & 19,52 & 26,20 & 8,77 & 36,64 & 26,93 & 24,72 & 4,54 & 19,85 & 23,33 \\
\hline & CVg $(\%)$ & 30,43 & 20,91 & 30,66 & 14,89 & 23,63 & 8,67 & 53,39 & 45,93 & 14,75 & 2,59 & 14,75 & 2,59 \\
\hline & Iv & 0,73 & 0,77 & 0,71 & 0,76 & 0,90 & 0,99 & 1,46 & 1,71 & 0,60 & 0,57 & 0,60 & 0,57 \\
\hline & $\mathrm{h}^{2}$ & 68,19 & 70,09 & 66,94 & 69,94 & 76,50 & 79,63 & 89,47 & 92,08 & 58,73 & 56,56 & 58,73 & 56,56 \\
\hline & $\mathrm{pV}(\%)$ & 6,90 & 0,12 & 2,75 & 0,13 & $<0,01$ & $<0,01$ & 0,37 & 1,88 & 0,37 & 1,88 & 1,53 & 1,50 \\
\hline
\end{tabular}

Continua ... 
Tabela 4. Continuação

\begin{tabular}{|c|c|c|c|c|c|c|c|c|c|c|c|c|c|}
\hline \multirow{2}{*}{ Exp. } & \multirow{2}{*}{ Parâmetro } & \multicolumn{2}{|c|}{ PT } & \multicolumn{2}{|c|}{ PC } & \multicolumn{2}{|c|}{ PMRC } & \multicolumn{2}{|c|}{ PR } & \multicolumn{2}{|c|}{ RI } & \multicolumn{2}{|c|}{ FORM } \\
\hline & & NT & $\mathbf{T}$ & NT & $T$ & NT & $\mathbf{T}$ & NT & $T$ & NT & $\mathbf{T}$ & NT & $\mathbf{T}$ \\
\hline \multirow{6}{*}{ IX } & Média & 1,72 & 1,01 & 1,31 & 1,01 & - & -3 & 43,22 & 13,12 & - & - & - & - \\
\hline & Cve (\%) & 75,10 & 3,43 & 79,07 & 3,33 & - & - & 16,54 & 11,65 & - & - & - & - \\
\hline & CVg $(\%)$ & 89,43 & 4,56 & 109,35 & 4,73 & - & - & 32,29 & 23,96 & - & - & - & - \\
\hline & Iv & 1,19 & 1,33 & 1,38 & 1,42 & - & - & 1,95 & 2,06 & - & - & - & - \\
\hline & $\mathrm{h}^{2}$ & 85,01 & 87,60 & 88,44 & 88,98 & - & - & 93,85 & 94,42 & - & - & - & - \\
\hline & $\mathrm{pV}(\%)$ & 0,08 & 0,02 & 0,02 & 0,01 & - & - & $<0,01$ & $<0,01$ & - & - & - & - \\
\hline \multirow{6}{*}{$\mathrm{X}$} & Média & 3,92 & 0,86 & 1,91 & 1,06 & $\begin{array}{l}\cdots \\
-\end{array}$ & - & 27,49 & 7,65 & - & $\begin{array}{l}\cdots \\
-\end{array}$ & - & - \\
\hline & Cve (\%) & 59,85 & 6,21 & 66,61 & 7,65 & - & - & 31,63 & 21,11 & - & - & - & - \\
\hline & CVg $(\%)$ & 7,82 & 2,34 & 33,84 & 4,06 & - & - & 41,22 & 28,74 & - & - & - & - \\
\hline & Iv & 0,13 & 0,38 & 0,51 & 0,53 & - & - & 1,30 & 1,36 & - & - & - & - \\
\hline & $h^{2}$ & 36,39 & 36,21 & 50,79 & 52,93 & - & - & 87,17 & 88,11 & - & - & - & - \\
\hline & $\mathrm{pV}(\%)$ & 41,15 & 12,95 & 3,86 & 3,02 & - & - & $<0,01$ & $<0,01$ & - & - & - & - \\
\hline \multirow{6}{*}{ XI } & Média & 24,61 & 3,23 & 15,29 & 3,15 & 230,00 & 0,36 & 29,37 & 1,49 & - & - & - & - \\
\hline & Cve (\%) & 30,87 & 11,55 & 39,33 & 17,77 & 29,66 & 5,07 & 47,47 & 5,50 & - & - & - & - \\
\hline & CVg $(\%)$ & 34,20 & 14,32 & 42,97 & 20,55 & 13,89 & 2,84 & 37,19 & 6,57 & - & - & - & - \\
\hline & Iv & 1,11 & 1,24 & 1,09 & 1,16 & 0,47 & 0,56 & 0,78 & 1,19 & - & - & - & - \\
\hline & $h^{2}$ & 83,08 & 86,00 & 82,68 & 84,24 & 46,74 & 55,67 & 71,05 & 85,09 & - & - & - & - \\
\hline & $\mathrm{pV}(\%)$ & $<0,01$ & $<0,01$ & $<0,01$ & $<0,01$ & $<0,01$ & $<0,01$ & $<0,01$ & $<0,01$ & - & - & - & - \\
\hline
\end{tabular}

${ }^{1}(\mathrm{NT})$ Dados que não sofreram transformação (data which were not transformed); ${ }^{2}$ X) Estimativas não apresentadas, pois a estimativa da variância genotípica apresentou valor negativo (estimates not presented because the estimated genetic variance showed a negative value); ${ }^{3}(-)$ variáveis não avaliadas no experimento (variables not measured in the experiment); $\mathrm{PT}=$ produtividade total de raízes, t/ha (total yield of roots, $\mathrm{t} / \mathrm{ha}$ ); $\mathrm{PC}=$ produtividade de raízes comerciais, $\mathrm{t} / \mathrm{ha}$ (yield of marketable roots, $\mathrm{t} / \mathrm{ha}$ ); $\mathrm{PMRC}=$ massa média de raízes comerciais, gramas (average mass of marketable roots, grams); $\mathrm{PR}=$ produtividade de ramas (yield of vines); $\mathrm{RI}=$ resistência das raízes a insetos de solo (resistance of roots agains soil insects); FORM= formato das raízes (shape of roots).

quando comparado com os coeficientes obtidos antes da transformação. Resultado similar foi verificado por Couto et al. (2009), Lúcio et al. (2010, 2011) que também encontraram grande redução no coeficiente de variação ambiental após a transformação Box-Cox. A redução das estimativas dos coeficientes de variação após a transformação dos dados é esperada, como consequência da mudança de escala. Os valores das estimativas de herdabilidade e do índice de variação não sofreram grande alteração após a transformação (Tabela 4), mostrando que não haveria mudanças nas conclusões em relação a estes parâmetros para o melhoramento genético em consequência da transformação. Quanto aos testes de hipótese para o efeito dos tratamentos, caso se considere o nível nominal de significância de $5 \%$, haveria mudanças nas conclusões para apenas em três casos, como consequência da transformação dos dados. Estas mudanças foram observadas nas características resistência das raízes ao ataque de patógenos no experimento IV, massa média de raízes comerciais no experimento VII e produtividade total no experimento VIII.

Hair et al. (2009) destacam que o atendimento da pressuposição de normalidade univariada contribui para a obtenção da distribuição normal multivariada. Para os resultados da normalidade multivariada, observaram-se melhorias nos valores de curtose, assimetria e para o teste Henze-Zirkler T, após o uso da transformação (Tabela 5). Os experimentos IV e XI, os quais apresentaram maiores desvios para a normalidade univariada, também foram os que tiveram problemas mais acentuados quanto à normalidade multivariada, com maiores estimativas para os testes de Mardia curtose, assimetria e para o teste Henze-Zirkler T.

Nos experimentos IV, IX, X e XI, embora os dados tenham se aproximado mais da distribuição normal multivariada, a hipótese de normalidade continuou sendo rejeitada pelo teste Henze-Zirkler
T (Tabela 5). Para os experimentos I, II, VI e VIII, a hipótese de normalidade multivariada passou a não ser rejeitada após a transformação. Toebe \& Cargnelutti Filho (2013), estudando oito características avaliadas em 13 ensaios de milho, verificaram a não rejeição da hipótese de distribuição normal multivariada após a transformação pela metodologia Box-Cox em todos os experimentos conduzidos. Assim, a transformação realizada individualmente nas variáveis representa uma alternativa mais simples, em comparação ao uso da transformação multivariada (Toebe \& Cargnelutti Filho, 2013). Os experimentos III, V e VII continuaram apresentando distribuição normal multivariada após a transformação. A não normalidade multivariada é algo muito comum, contudo nem sempre ela é identificada, já que a maioria dos pesquisadores não atenta para a sua importância. Desvios na normalidade multivariada podem ocasionar equívocos nas inferências de testes estatísticos 
Tabela 5. Valores dos testes de normalidade multivariada considerando os dados não transformados (NT) e transformados (T) pela metodologia Box-Cox em 11 experimentos de batata-doce \{values of multivariate normality tests considering no transformed data (NT) and transformed (T) by the Box-Cox method in 11 experiments of sweet potato $\}$. Diamantina, UFVJM, 2015.

\begin{tabular}{|c|c|c|c|c|}
\hline Ensaios & & Mardia Curtose & Mardia Simetria & Henze-Zirkler T \\
\hline \multirow{2}{*}{ I } & NT & $0,96^{\mathrm{ns}}$ & $97,60^{* *}$ & $2,96^{* *}$ \\
\hline & $\mathrm{T}$ & $0,28^{\mathrm{ns}}$ & $81,00^{*}$ & $1,16^{\mathrm{ns}}$ \\
\hline \multirow{2}{*}{ II } & NT & $2,20^{*}$ & $114,00^{* *}$ & $4,77^{* *}$ \\
\hline & $\mathrm{T}$ & $1,00^{\mathrm{ns}}$ & $85,00^{*}$ & $1,86^{\mathrm{ns}}$ \\
\hline \multirow{2}{*}{ III } & NT & $0,44^{\mathrm{ns}}$ & $95,49^{* *}$ & $1,45^{\mathrm{ns}}$ \\
\hline & $\mathrm{T}$ & $0,01^{\mathrm{ns}}$ & $80,71^{*}$ & $1,58^{\mathrm{ns}}$ \\
\hline \multirow{2}{*}{ IV } & NT & $13,24^{* * *}$ & $530,00^{* *}$ & $20,91^{* *}$ \\
\hline & $\mathrm{T}$ & $2,99^{* *}$ & $113,30^{* *}$ & $4,33^{* *}$ \\
\hline \multirow{2}{*}{ V } & NT & $0,77^{\mathrm{ns}}$ & $87,79^{* *}$ & $0,91^{\mathrm{ns}}$ \\
\hline & $\mathrm{T}$ & $-0,21^{\mathrm{ns}}$ & $65,42^{\text {ns }}$ & $0,82^{\text {ns }}$ \\
\hline \multirow{2}{*}{ VI } & NT & $0,12^{\mathrm{ns}}$ & $79,66^{*}$ & $2,98^{* * *}$ \\
\hline & $\mathrm{T}$ & $0,5^{\mathrm{ns}}$ & $65,03^{\text {ns }}$ & $0,91^{\mathrm{ns}}$ \\
\hline \multirow{2}{*}{ VII } & NT & $1,16^{\mathrm{ns}}$ & $101,4^{*}$ & $1,03^{\mathrm{ns}}$ \\
\hline & $\mathrm{T}$ & $0,66^{\mathrm{ns}}$ & $57,9^{\text {ns }}$ & $0,45^{\mathrm{ns}}$ \\
\hline \multirow{2}{*}{ VIII } & NT & $1,14^{\mathrm{ns}}$ & $96,09^{* *}$ & $3,66^{* * *}$ \\
\hline & $\mathrm{T}$ & $4,29^{* *}$ & $171,00^{* *}$ & $1,77^{\mathrm{ns}}$ \\
\hline \multirow{2}{*}{ IX } & NT & $4,72^{* *}$ & $78,29^{* * *}$ & $5,05^{* * *}$ \\
\hline & $\mathrm{T}$ & $-0,54^{\mathrm{ns}}$ & $23,89^{* *}$ & $3,05^{* *}$ \\
\hline \multirow{2}{*}{$\mathrm{X}$} & NT & $11,33^{* *}$ & $156,10^{* *}$ & $5,74^{* *}$ \\
\hline & $\mathrm{T}$ & $4,07^{* *}$ & $25,50^{* *}$ & $2,40^{*}$ \\
\hline \multirow{2}{*}{ XI } & NT & $21,78^{* *}$ & $459,40^{* *}$ & $11,85^{* *}$ \\
\hline & $\mathrm{T}$ & $4,62^{* *}$ & $93,91^{* *}$ & $5,33^{* *}$ \\
\hline
\end{tabular}

**, ${ }^{*} \mathrm{e}^{\mathrm{ns}}$ referem-se à significância a $1 \%, 5 \%$ e não significativo a $5 \%$ respectivamente ${ }^{* *}$, *, and ${ }^{\text {ns }}$ correspond to significance of $1 \%, 5 \%$ and not significant at the $5 \%$ respectively).

da análise multivariada e análises de modelos mistos multi-característica.

Logo, observou-se que nesses experimentos de batata-doce não houve uma transformação específica que fosse ideal para cada uma das variáveis estudadas. Além disso, nem sempre é necessário o uso da transformação de dados. A transformação Box-Cox melhorou a homocedasticidade e normalidade uni e multivariada, porém, nem sempre as hipóteses de homocedasticidade e normalidade deixaram de ser rejeitadas após a transformação. A transformação Box-Cox praticamente não alterou as estimativas de herdabilidade do índice de variação e das conclusões do teste $F$ para o efeito de tratamento na maioria dos casos; no entanto, promoveu redu-
FERNANDES JSC; FIGUEIREDO JA; NUNES UR; NEIVA IP. 2009. Selection of sweet potato clones for the region Alto Vale do Jequitinhonha. Horticultura brasileira 27: 389-393.

ANDRADE JÚNIOR VC; VIANA DJS; PINTO NAVD; RIBEIRO KG; PEREIRA RC; NEIVA IP; AZEVEDO AM; ANDRADE PCR. 2012. Características produtivas e qualitativas de ramas e raízes de batata-doce. Horticultura Brasileira 30: 584-589.

AZEVEDO AM; ANDRADE JÚNIOR VC; FERNANDES JSC; PEDROSA CE; OLIVEIRA CM. 2015. Desempenho agronômico e parâmetros genéticos em genótipos de batata-doce. Horticultura Brasileira 33: 084-090.

AZEVEDO AM; VIANA DJS; ANDRADE JÚNIOR VC; ELSAYED AYAM; PEDROSA CE; NEIVA IP; FIGUEIREDO JA. 2014. Influence of harvest time and cultivation sites on the productivity and quality of sweet potato. Horticultura Brasileira 32: 8-14.

AZEVEDO SM; MALUF WR; SILVEIRA MA; FREITAS JA. 2002. Reação de clones de batata-doce aos insetos de solo. Ciência e Agrotecnologia 26: 545-549.

BANZATTO DA.; KRONKA SN. 2013. Experimentação Agrícola. Jaboticabal: FUNEP. 237p.

BOX GEP; COX DR. 1964. An analysis of transformations. Journal of the Royal Society 26: $211-252$.

CANTELMO NF; FERREIRA DF; 2007. Desempenho de testes de normalidade multivariados avaliado por simulação monte carlo. Ciência e Agrotecnologia 31: 16301636.

CARDOSO AD; VIANA AES; MATSUMOTO SN; BONFIM NETO H; KHOURI CR; MELO, TL. 2007. Características físicas e sensoriais de clones de batata-doce. Ciência e Agrotecnologia 31: 1760-1765.

CARDOSO AD; VIANA AES; RAMOS PAS; MATSUMOTO SN; AMARAL CLF; SEDIYAMA T; MORAIS OM. 2005. Avaliação de batata-doce em Vitória da Conquista. Horticultura Brasileira 23: 911914.

CAVALCANTE JT; FERREIRA PV; SOARES L. 2003. Avaliação de clones de batata-doce (Ipomoea batatas (L.) Lam.) em Rio Largo Alagoas. Magistra 15: 13-17.

CAVALCANTE JT; FERREIRA PV; SOARES L; BORGES V; SILVA PP; SILVA JW. 2006. Análise de trilha em caracteres de rendimento de clones de batata-doce (Ipomoea batatas (L.) Lam). Acta Scientiarum 28: 261-266.

CHUNG SH; PEARN WL; YANG YS. 2007. A comparison of two methods for transforming non-normal manufacturing data. International Journal of Advanced Manufacturing Technology 31: 957-968.

COUTO MRM; LÚCIO AD; LOPES SJ; CARPES RH. 2009. Transformação de dados em experimentos com abobrinha italiana em ambiente protegido. Ciência Rural 39: 17011707.

CRUZ CD. 2013. GENES - a software package for analysis in experimental statistics and 
quantitative genetics. Acta Scientiarum 35: 271-276.

CRUZ CD; REGAZZI AJ; CARNEIRO PCS. 2012. Modelos biométricos aplicados ao melhoramento genético. Viçosa: Editora UFV. $514 \mathrm{p}$.

CUSTÓDIO TN; BARBIN D. 2009. Modelos de predição para sobrevivência de plantas de Eucalyptus grandis. Ciência e Agrotecnologia 33: 1948-1952.

FILGUEIRA FAR. 2008. Novo manual de olericultura: Agrotecnologia moderna na produção e comercialização de hortaliças. Viçosa: Editora UFV. 402p.

FREITAS AR; PRESOTTI CV; TORAL FLB. 2005. Alternativas de análises em dados de medidas repetidas de bovinos de corte. Revista Brasileira de Zootecnia 34: 2233-2244.

FRENI G.; MANNINA G. 2012. Uncertainty estimation of a complex water quality model: The influence of Box-Cox transformation on Bayesian approaches and comparison with a non-Bayesian method. Physics and Chemistry of the Earth 42: 31-41.

GONÇALVES NETO AC; MALUF WR; GOMES LAA; GONÇALVES RJS; SILVA VF; LASMAR A. 2011. Aptidões de genótipos de batata-doce para consumo humano, produção de etanol e alimentação animal. Pesquisa Agropecuária Brasileira 46: 1513-1520.

GONÇALVES NETO AC; MALUF WR; GOMES LAA; MACIEL GM; FERREIRA RPD; CARVALHO RC. 2012. Correlação entre caracteres e estimação de parâmetros populacionais para batata-doce. Horticultura Brasileira 30: 713-719.

HAIR JF; BLACK WC; BABIN BJ; ANDERSON
RE; TATHAM RL. 2009. Análise multivariada de dados. 6. ed. Porto Alegre: Bookman. 688p.

LÚCIO AD; COUTO MRM; LOPES SJ; STORCK L. 2011. Transformação box-cox em experimentos com pimentão em ambiente protegido. Horticultura Brasileira 29: 38-42.

LÚCIO AD; COUTO MRM; TREVISAN JN; MARTINS GAK; LOPES SJ. 2010. Excesso de zeros nas variáveis observadas: estudo de caso em experimento com brócolis. Bragantia 69: 1035-1046.

MARCHESE A; MALUF WR; GONÇALVES NETO AC; GONÇALVES RDS; GOMES LAA. 2010. Seleção de clones de batata-doce resistentes a Meloidogyne incognita raça 1. Pesquisa Agropecuária Brasileira, 45: 997-1004.

MARDIA KV. 1970. Measures of multivariate skewness and kurtosis with applications. Biometrika 57: 519-530.

MASSAROTO JA; GOMES LAA; MALUF WR; SILVA RR; GOMES ARVA. 2010. Reação de clones de batata-doce a Meloidogyne incognita raça 1. Revista de Ciências Agroambientais 8: $1-8$.

MECKLIN CJ. 2004. An appraisal and bibliography of test for multivariate normality. International Statistical Review 72: 123-138.

MELOUN M; SANKA M; NEMEC P; KRITCKOVAS; KUPK, K. 2005. The analysis of soil cores polluted with certain metals using the Box-Cox transformation. Environmental Pollution 137: 273-280.

MOREIRA JN; QUEIROGA RC; SOUSA JÚNIOR AJL; SANTOS MA. 2011. Caracteres morfofisiológicos e produtivos de cultivares de batata-doce, em Mossoró, RN. Revista Verde 6: 161-167.

PIMENTEL-GOMES F. 2009. Curso de estatística experimental. FEALQ: Piracicaba. 451p.

RESENDE MDV. 2007. Matemática e estatística na análise de experimentos e no melhoramento genético. Colombo: Embrapa Florestas. 561p.

RIBEIRO AC; GUIMARÃES PTG; ALVAREZ VVH. 1999. Recomendações para o uso de corretivos e fertilizantes em Minas Gerais: $5^{a}$ aproximação. Viçosa: Comissão de Fertilidade do solo do Estado de Minas Gerais. 180 p.

SANTOS AC; FERREIRA DF. 2003. Definição do tamanho amostral usando simulação Monte Carlo para o teste de normalidade baseado em assimetria e curtose: I. Abordagem univariada. Ciência e Agrotecnologia 27: 432-437.

TOEBEM; CARGNELUTTI FILHOA. 2013. Não normalidade multivariada e multicolinearidade na análise de trilha em milho. Pesquisa Agropecuária Brasileira 48: 466-477.

TRUXILLO C. 2005. Multivariate statistical methods: Practical research applications. Cary: SAS Institute.

VIANA DJSV; ANDRADE JUNIOR VC; RIBEIRO GK; PINTO NAVD; NEIVA IP; FIGUEIREDO JA; LEMOS VT; PEDROSA CE; AZEVEDO AM. 2011. Potencial de silagens de ramas de batata-doce para alimentação animal. Ciência Rural 41: 14661471.

XU W; LI W; SONG D. 2013. Testing normality in mixed models using a transformation method. Stat Papers 84: 54:71.

YANG R; YI N; XU S. 2006. Box-Cox transformation for QTL mapping. Genetica 128:133-143. 